\title{
ECOSYSTEM STABILITY AND BIFURCATION IN THE LIGHT OF ADAPTABILITY THEORY
}

\author{
Michael Conrad \\ Department of Computer and Communication Sciences \\ University of Michigan \\ Ann Arbor, Michigan 48109
}

\section{INTRODUCTION}

In the author's laboratory an experiment of the following type is performed. Replicas of "jar microecosystems" are prepared as identically as possible from undefined pond material (water, bottom matter). Ensembles of these replicas are then cultured in incubators under varying degrees of statistical uncertainty and physical stress. Such microecosystems, like all ecosystems, undergo a sequence of changes (called succession) until they reach a climax form of organization. This is a form of organization which appears to be stable in the sense that it persists for a relatively long time. A necessary condition for such persistence is that the system is somehow capable of either dissipating or absorbing without visible effect the perturbations to which it is being subjected; after all, if it were not capable of doing this it would continue to change. By choosing the degree of environmental uncertainty it is thus possible to prepare (through the culturing process) ecosystems capable of absorbing or dissipating at minimum this degree of environmental uncertainty.

The ability to cope with (absorb or dissipate) environmental uncertainty will be called adaptability. Thus, the imposed uncertainty of the culturing environment provides an operational definition of minimum adaptability. Once systems with defined minimum adaptabilities are prepared, many questions might be asked. For example, does actual adaptability tend to decrease in the direction of the minimum possible? What is the relation between adaptability and the stability of succession? What is the relation to the spectrum of particular forms of adaptability (e.g., genetic, organismic, populational) and to patterns of community organization? The intention of this paper, however, is not to consider the experimental aspect of this problem, but rather to describe a formalism which makes it possible to approach complete, self-sustaining biological systems of the above type theoretically, in particular to analyze the complex of adaptabilities underlying their dynamics.

The essence of this formalism (hierarchical adaptability theory) is an entropy theory analysis of the state-to-state behavior of ecosystems in which the state specifications are structured in a way which reflects the hierarchical and compartmental organization of complete, self-sustaining biological systems (including their environment). After briefly reviewing this formalism (cf. also Conrad ${ }^{1-7}$ ), we shall state and prove the bootstrap principle of hierarchical adaptability theory (that the assumption of structured descriptions is self-justifying). The bootstrap principle underlies the major conclusion, viz., that as the dynamics of any particular level in an ecosystem appears more autonomous (i.e., more amenable to 
description without reference to variables associated with other levels), the contribution of adaptabilities at other levels in general becomes more important as support for these dynamics. This makes it possible to cross-correlate the elements of hierarchical adaptability theory with concepts deriving from dynamical styles of analysis and therefore to make some statements about the hidden biology underlying ecosystem stability and bifurcation.

\section{Formalism of Hierarchical Adaptability Thêror}

\section{Structured State Specifications}

The ecosystem is supposed to consist of a living (biotic) part and a physical environment, each with a finite set of distinguishable states observed on a discrete time scale. (These assumptions simplify some technical problems, but could be dropped.) The state of both the living part (community) and the environment is furthermore supposed to be adequately specifiable in terms of a finite set of observable properties. In the case of the community these properties include: species composition, foodweb structure, number of organisms in each species, locations of each organism, physiological state of each organism, pattern of gene activation and also genotype (DNA base sequence) of each organism. In the case of the environment the physical space is divided into local regions and the state of each of these regions (excluding that portion occupied by living matter) specified in terms of macroscopic variables (e.g., temperature, pressure, mole numbers, other mechanical forces).

The above state specifications can be thought of as structured descriptions of ecological systems. In the case of the community this structuring corresponds to the apparent organizational structure, i.e., to the levels of organization in biological systems, with more or less identifiable units (or compartments) at the various levels. Thus, the community can be considered as a unit, populations as subunits of the community, organisms as subunits of populations, and genome and phenome (all of the organism aside from the genome) as subunits of the organism. Species composition and foodweb organization are thus partial states (or partial characterizations) of the community, without reference to species, organisms, or other lower level compartments. Number and locations of organisms are partial states of species, again without reference to lower level subunits. For the present purposes, the pattern of gene activation can be thought of as the partial state of the organism in terms of phenome and genome and physiological state and DNA sequence as complete states of the phenome and genome. The procedure could be carried further, by increasing the number of levels and decreasing the number of properties required to characterize compartments at each level (assuming that a complete set of properties has been chosen in the first place).

The above correspondence between structured state descriptions and hierarchical and compartmental structure can be made formal by defining complete states as many-tuples of partial states. Thus,

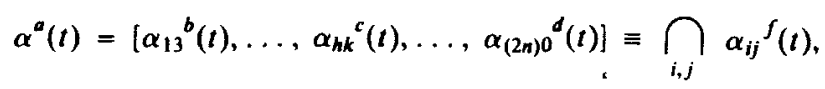


where the $\alpha^{u}$ are complete states of the community, the $\alpha_{i j}{ }^{\prime}$ are partial states of compartment $i$ at level $j$; level 3 can be taken as the community level (and there is only one community), level 2 can be taken as the population level, level 1 as the organism level, level 0 as either the genome or phenome level (in which case the states are complete), and the re-representation is possible for some choice of superscripts. (Actually, since matter cycles in an ecosystem and since organisms are born and die, it is necessary to define a reference structure with a very large number of compartments, some "unborn" or "dead" at any given time. ${ }^{3}$ ) Also, for the environment,

$$
\beta^{a}(t)=\left[\beta_{i 0}^{b}(t), \ldots, \beta_{m 0}^{c}(t)\right] \equiv \bigcap_{h} \beta_{h 0^{8}(t),}
$$

where the $b^{v}$ are complete states of the environment, the $\beta_{i 0}{ }^{s}$ are complete states of region $i$ at level 0 , the convention is that all compartments are at level 0 , and the re-representation is possible for some choice of superscripts.

Later it is shown that structurability of community descriptions is a selfjustifying assumption.

\section{Transition Schemes and Transition Scheme Entropies}

Imagine a large number of replicas of microecosystems, all prepared as identically as possible and undergoing state to state transitions in time. One may suppose that for any given time, starting from any given initial state, there would exist in principle a set of transition probabilities

$$
\Omega=\left\{p\left[\alpha^{u}(t+1), \beta^{v}(t+1) \mid \alpha^{\prime}(t), \beta^{s}(t)\right]\right\} .
$$

For the community and environment separately this implies the partial schemes

$$
\begin{aligned}
\omega & =\left\{p\left[\alpha^{\mu}(t+1) \mid \alpha^{\prime}(t), \beta^{s}(t)\right]\right\} \\
\omega^{\prime} & =\left\{p\left[\beta^{v}(t+1) \mid \alpha^{\prime}(t), \beta^{s}(t)\right]\right\}
\end{aligned}
$$

where the prime notation distinguishes the environment scheme. On these partial schemes entropies may be defined, e.g.,

$H\left(\omega^{\prime}\right)=-\Sigma p\left[\alpha^{\prime}(t), \beta^{s}(t)\right] p\left[\beta^{t}(t+1) \mid \alpha^{\prime}(t), \beta^{s}(t)\right] \times$

$$
\log p\left[\beta^{v}(t+1) \mid \alpha^{\prime}(t), \beta^{s}(t)\right]
$$

and

$$
H\left(\omega \mid \omega^{\prime}\right)=-\Sigma p\left[\alpha^{r}(t), \beta^{s}(t), \beta^{v}(t+1)\right] p\left[\alpha^{u}(t+1) \mid \alpha^{r}(t), \beta^{s}(t), \beta^{v}(t+1)\right] \times
$$

$$
\log p\left[\alpha^{u}(t+1) \mid \alpha^{r}(t), \beta^{s}(t), \beta^{v}(t+1)\right],
$$

where indices run over all sums and corresponding entropies may be written for $H(\omega)$ and $H\left(\omega^{\prime} \mid \omega\right)$. In analogy to a well known identity of entropy theory,

$$
H(\omega)-H\left(\omega \mid \omega^{\prime}\right)+H\left(\omega^{\prime} \mid \omega\right)=H\left(\omega^{\prime}\right),
$$

which can easily be proved for this generalized case by substituting the transition scheme definitions. Equation 7 is always true and expresses what is in fact a 
tautological relation between the observed statistical properties of the community and the observed statistical properties of the environment.

\section{Operational Definition of Adaptability}

In order to use the foregoing to develop a definition of adaptability it is necessary for the terms on the left-hand side of Equation 7 to represent potentialities, independent of the particular statistical character of the environment. This can be done by varying over all possible environments, looking for the most uncertain environment compatible with the system remaining alive. If this environment has transition scheme $\omega^{\prime}$, Equation 7 can be expressed as the inequality

$$
H(\hat{\omega})-H\left(\hat{\omega} \mid \hat{\omega}^{\prime}\right)+H\left(\hat{\omega}^{\prime} \mid \hat{\omega}\right) \geqq H\left(\omega^{\prime}\right)
$$

where $H(\hat{\omega})$ is the potential uncertainty of the community, $H\left(\hat{\omega} \mid \hat{\omega}^{\prime}\right)$ reflects the potential ability to anticipate the environment, $H\left(\hat{\omega}^{\prime} \mid \hat{\omega}\right)$ reflects the potential indifference to the environment, and $\omega^{\prime}$ is the transition scheme of the actual environment. The entire left-hand side of Equation 8 will be called the maximal adaptability of the biological system. Actually the condition that the system undergo complete demise is a rather strong one if the biological system is a complete community (as opposed to, say, a single population or organism) and in general it is more reasonable to replace it by a weaker condition, e.g., that the community not be pushed out of a given edaphic climax (in which case maximal adaptability is defined only for this climax) or that it not exhibit more than some predefined degree of decrement in biomass. The latter condition introduces a certain arbitrariness into the definition, but this can be removed to the extent that the relative adaptabilities (of at least initially identical systems) can be expected to be properly ordered.

It is possible to replace the above definition of (maximal) adaptability by a definition of another quantity (minimal adaptability) which extends the idea of relative ordering in the sense that it requires no measurement of cell death and which at the same time may quite reasonably be expected to provide an index of actual adaptability. This definition, however, requires a postulate, viz., the culture procedure postulate (really the fundamental postulate of microbiology since the work of Koch). To express this, first consider an environment with transition scheme $\bar{\omega}^{\prime}$. Equation 8 can then be written, for this special case, as

$$
H(\bar{\omega})-H\left(\bar{\omega} \mid \bar{\omega}^{\prime}\right)+H\left(\bar{\omega}^{\prime} \mid \bar{\omega}\right)=H\left(\bar{\omega}^{\prime}\right),
$$

where $\bar{\omega}$ is the transition scheme of the biota in this environment. If the community is allowed to culture in an environment $\bar{\omega}^{\prime}$ for a very long time (in practice, long relative to successional time scales) the left-hand side of Equation 9 will be called the minimal adaptability of the community. The culture procedure postulate is: Minimal adaptability is an index of maximal adaptibility in the sense that a community with higher minimal adaptability will suffer less damage (as measured by biomass decrement) than a community with lower minimal adaptability when both communities are exposed to an environment more uncertain than either culturing environment. (The condition should also be added that the two systems are initially 
identical and that the culturing environment is as nearly as identical as possible except for the difference in uncertainty.)

The culture procedure postulate is based on the idea that never used components of adaptability (larger than necessary behavioral repertories, better than necessary ability to anticipate, more than necessary indifference) always tend to atrophy in the succession or evolution process. Thus Equation 8 can be replaced by

$$
H(\hat{\omega})-H\left(\hat{\omega} \mid \hat{\omega}^{\prime}\right)+H\left(\hat{\omega}^{\prime} \mid \hat{\omega}\right) \rightarrow H\left(\omega^{\prime}\right),
$$

where the arrow indicates a tendency for the adaptability to decrease in the direction of the actual uncertainty of the environment and thus to decrease in the direction of minimal adaptability (if $\omega^{\prime}=\bar{\omega}^{\prime}$ ). Thus, the arrow can be taken to mean that in the course of succession or evolution adaptability decreases in the sense that the amount of biomass decrement following on an increase in the uncertainty of the environment would be greater.

The culture procedure postulate is a local postulate since only systems which are in all respects similar except for the uncertainty of the culturing environment are being compared.

\section{Structure of Adaptability}

The components of adaptability may be further decomposed, using the structured state descriptions expressed in Equations 1 and 2. To this the transition scheme of the community is written in terms of the joint occurrence of all possible subcompartment transitions

$$
\omega=\left\{p\left[\bigcap_{i, j} \alpha_{i j}^{d}(t+1) \mid \bigcap_{i, j} \alpha_{i j}^{f}(t), \bigcap_{h} \beta_{h 0^{8}}(t)\right]\right\} .
$$

Defining local transition schemes for each compartment

$$
\omega_{p q}=\left\{p\left[\alpha_{p q}{ }^{u}(t+1) \mid \bigcap_{i, j} \alpha_{i j}^{f}(t), \bigcap_{h} \beta_{h 0^{8}}(t)\right]\right\}
$$

Equation 11 can be expressed as

$$
\omega=\prod_{i, j} \omega_{i j}
$$

where care should be taken to realize that this is not a bona fide product, but rather shorthand for the composition of all (or some set) of local schemes. Similarly, the transition scheme of the environment can be expressed in terms of a "product" of transition schemes for local regions

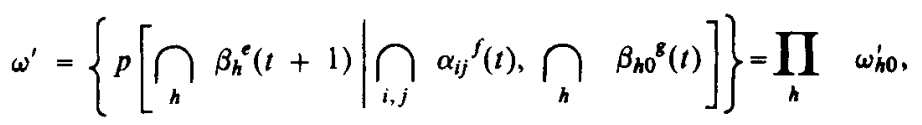

where

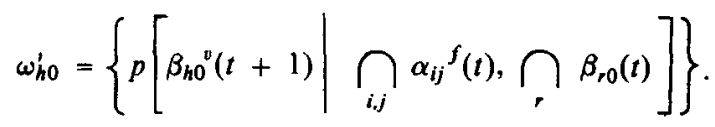


Substituting the above compositions into Equation 10, we find

$$
\begin{aligned}
& H\left(\prod_{i, j} \hat{\omega}_{i j}\right)-H\left(\prod_{i, j} \hat{\omega}_{i j} \mid \prod_{h} \hat{\omega}_{h 0}^{\prime}\right)+H\left(\prod_{h} \hat{\omega}_{h 0}^{\prime} \mid \prod_{i, j} \hat{\omega}_{i j}\right) \\
& \rightarrow H\left(\prod_{h} \omega_{h 0}^{\prime}\right) .
\end{aligned}
$$

Each of the terms in the above equation may be expressed (using identities analogous to Equation 7) in terms of sums of unconditional and conditional entropies. However, since there are many possible expansions of this type, it is convenient to choose a single canonical form which puts all the subcompartments on an equal footing. To do this, define the effective entropy, $H_{e}\left(\omega_{i j}\right)$ as the sum of the unconditional and all possible conditional entropies of compartment $i$ at level $j$, but normalized by normalizing the linear combination of all possible expansions (for an example, see Equations 19a,b,c). With this convention Equation 16 can be written

$$
\sum_{i, j} H_{e}\left(\hat{\omega}_{i j}\right)-\sum_{i, j} H_{e}\left(\hat{\omega}_{i j} \mid \prod_{h} \hat{\omega}_{h 0}^{\prime}\right)+\sum_{h} H_{e}\left(\hat{\omega}_{h 0}^{\prime} \mid \prod_{i, j} \hat{\omega}_{i j}\right) \rightarrow \sum_{h} H_{e}\left(\omega_{h 0}^{\prime}\right) .
$$

Each term in this equation can be identified with a possible mechanism of adaptability. Thus the unconditional parts of the effective entropies express the indeterminacy of the genetic endowment of an organism, phenotypic plasticity (e.g., morphological, physiological, behavioral), developmental plasticity (i.e., alternate expressibility of the genome), numerical and topographical plasticity of populations, and so forth. The conditional terms express the degree of independence among these different plasticities, the anticipation terms reflect the effectiveness with which they are used, and the indifference term expresses the niche structure (both spatial and physiochemical). The equation itself formally expresses a principle of compensation among adaptabilities, viz., that change in the adaptability of one subsystem tends to be compensated by opposite changes in the adaptability of other subsystems, at the same or different levels, or by opposite changes in the indifference to the environment. The costs and advantages of such compensations depend on factors such as the morphological organization of the system and the time scale of environmental disturbance. In this sense the spectrum of adaptabilities in nature can be regarded as an optimization problem.

Equation 17 could also be written in terms of the $\bar{\omega}_{i j}$, in which case the compensations are among minimum adaptabilities. In what follows the argument could be expressed either in terms of the $\hat{\omega}_{i j}$ or the $\bar{\omega}_{i j}$.

\section{Bootstrap Principle of Adaptability Theory}

The bootstrap principle of adaptability theory is: The assumption of the hierarchical and compartmental structurability of the state description of biological systems is self-justifying within the framework of adaptability theory in the sense that such structurability is a necessary condition for efficient adaptability. By efficiency of adaptability is here meant the relative cost of adaptability in terms of energy 
or in terms of interference with biological function (which ultimately appears in terms of an energy cost). Thus, suppose the morphological structure of an organism is extremely complex. Developmental and genetic plasticity would be relatively more costly in this case since genetic variations are more likely to be disruptive and alternative modes of development would require the accumulation and maintenance of much more genetic information. As another example, suppose that modifications of organism behavior are coordinated to morphological modifications involving growth processes. This clearly increases the energy that must be expended to maintain behavioral plasticity. More generally stated: Any increase in the extent of modification required to achieve a given degree of adaptability increases the biological costs (ultimately measurable in terms of energy utilization) associated with that adaptability. This generally is in fact implicit in the culture procedure postulate.

Given the above, the bootstrap principle is fairly easy to prove. To make things concrete, consider the simple, three compartment decomposition of a single organism (organism one at level one, with genome and phenome compartments one and two at level zero). For the behavioral uncertainty component of adaptability

$$
H\left(\hat{\omega}_{10} \hat{\omega}_{20} \hat{\omega}_{11}\right)=H_{e}\left(\hat{\omega}_{10}\right)+H_{e}\left(\hat{\omega}_{20}\right)+H_{e}\left(\hat{\omega}_{11}\right),
$$

where

$$
\begin{aligned}
& H_{e}\left(\hat{\omega}_{10}\right)=\frac{1}{3}\left\{H\left(\hat{\omega}_{10}\right)+\frac{1}{2} H\left(\hat{\omega}_{10} \mid \hat{\omega}_{20}\right)+\frac{1}{2} H\left(\hat{\omega}_{10} \mid \hat{\omega}_{11}\right)+H\left(\hat{\omega}_{10} \mid \hat{\omega}_{20} \hat{\omega}_{11}\right)\right\} \\
& H_{e}\left(\hat{\omega}_{20}\right)=\frac{1}{3}\left\{H\left(\hat{\omega}_{20}\right)+\frac{1}{2} H\left(\hat{\omega}_{20} \mid \hat{\omega}_{10}\right)+\frac{1}{2} H\left(\hat{\omega}_{20} \mid \hat{\omega}_{11}\right)+H\left(\hat{\omega}_{20} \mid \hat{\omega}_{10} \hat{\omega}_{11}\right)\right\} \\
& H_{e}\left(\hat{\omega}_{11}\right)=\frac{1}{3}\left\{H\left(\hat{\omega}_{11}\right)+\frac{1}{2} H\left(\hat{\omega}_{11} \mid \hat{\omega}_{10}\right)+\frac{1}{2} H\left(\hat{\omega}_{11} \mid \hat{\omega}_{20}\right)+H\left(\hat{\omega}_{11} \mid \hat{\omega}_{10} \hat{\omega}_{20}\right)\right\} .
\end{aligned}
$$

Each effective entropy consists of an unconditioned modifiability term (to be called the modifiability term) and a number of conditioned modifiability terms (to be called the independence terms). For given modifiabilities the effective entropies are largest when each of the conditioned terms are equal and equal to the modifiability term (since they cannot be larger). Thus, the conditions for a maximum uncertainty component of adaptability for given observable modifiabilities are

$$
\begin{aligned}
& H\left(\hat{\omega}_{10}\right)=H\left(\hat{\omega}_{10} \mid \hat{\omega}_{20}\right)=H\left(\hat{\omega}_{10} \mid \hat{\omega}_{11}\right)=H\left(\hat{\omega}_{10} \mid \hat{\omega}_{10} \hat{\omega}_{11}\right), \\
& H\left(\hat{\omega}_{20}\right)=H\left(\hat{\omega}_{20} \mid \hat{\omega}_{10}\right)=H\left(\hat{\omega}_{20} \mid \hat{\omega}_{11}\right)=H\left(\hat{\omega}_{20} \mid \hat{\omega}_{10} \hat{\omega}_{11}\right), \\
& H\left(\hat{\omega}_{11}\right)=H\left(\hat{\omega}_{11} \mid \hat{\omega}_{10}\right)=H\left(\hat{\omega}_{11} \mid \hat{\omega}_{20}\right)=H\left(\hat{\omega}_{10} \mid \hat{\omega}_{10} \hat{\omega}_{20}\right),
\end{aligned}
$$

where $H\left(\hat{\omega}_{10}\right), H\left(\hat{\omega}_{20}\right)$, and $H\left(\hat{\omega}_{11}\right)$ are the observable modifiabilities. To the extent that these conditions are not realized, the observable modifiabilities of different compartments will be correlated (because the independence terms are relatively small) and therefore the extent of modification required to achieve a given degree of adaptability would increase. However, according to the above version of the culture procedure postulate, this increases the costs of adaptability. In short, a necessary condition for efficient adaptability is that the independence terms are not small relative to their modifiability term. To the extent that this is true, however, it is also true that compartmental (and level) structure will be a necessary condition for efficient adaptability. In this sense the initial assumption of 
hierarchical and compartmental structure is self-justifying from the standpoint of adaptability theory.

The above conclusion clearly generalizes for an arbitrary number of compartments and levels and therefore for the community as a whole. This does not mean that the condition of maximum independence of compartments is ever obtained. In general this is impossible and in many cases strong correlation is inherent in fundamental biological constraints. The most important example is the relation between genome and phenome. Specification of the phenome completely determines the genome, but specification of the genome only partially determines the phenome. This is because a given genome only specifies a repertoire of possible phenome states, but in general any genetic change (other than certain degenerate changes) modifies this repertoire. This constraint is clearly fundamental since it is the basis of evolution. However, to the extent that the effects of environmental disturbance can be prevented from ramifying to more and more compartments, the absorption or dissipation of these disturbances will be less costly.

\section{Dynamical Autonomy and the Role of UNREPRESENTED ADAPTABILITIES}

\section{Degree of Autonomy}

A compartment (or level) will be said to be autonomous to the extent that its behavior is independent of other compartments (or levels). From the standpoint of the transition scheme $\omega_{i j}$, a compartment or level is autonomous to the extent that the transition probabilities need not be conditioned on other compartments or levels. From the standpoint of the scheme entropies, a compartment or level is independent to the extent that its independence terms are large relative to the modifiability term. The degree of autonomy can be expressed by the ratio

$$
\xi_{i j}=H_{e}\left(\hat{\omega}_{i j}\right) / H\left(\hat{\omega}_{i j}\right),
$$

where compartment $(i, j)$ is completely autonomous if $\xi_{i j}=1$ and not autonomous if $\xi_{i j}<1$ (since the effective entropy is less than the unnormalized modifiability).

\section{Autonomy and Predictability (for Discrete Time, Finite State Dynamical Systems)}

If compartment $(i, j)$ is completely autonomous $\left(\xi_{i j}=1\right), \hat{\omega}_{i j}$ is as good a predictor of the behavior of this compartment as the global community scheme' $\hat{\omega}$. In this case only properties (variables) of the compartment need enter into the predictor. If $\xi_{i j}<1$, the "law will be broken," with the degree of breaking depending on the degree of autonomy (or degree of dependence). In this case variables from other compartments must be added to the predictor to restore its predictive power. (If the modifiability is high and the anticipation term low, reflecting good anticipation, a still better predictor could of course be constructed by including environmental variables.)

The predictor here has been written in the form of a transition scheme (cf. 
Equations 4a \& 13). The data provided by this setup are: a set of states of the community, a set of inputs (states of the environment), a set of states of the compartment in question (which may be the community or a subunit of the community), the probability distribution of initial states of the community and environment, and a rule (assumed for the sake of generality to be probabilistic) for determining the state of the compartment at the next instant of time. This is the same information that would be necessary to define the compartment as an automaton (since, according to Equation 1, specification of the state of the community automatically entails specification of the states of all its subcompartments). Thus the predictor can be interpreted as the transition function (or rule) governing the behavior of the compartment, viewed as an automaton (actually semiautomaton, or automaton without output). A necessary condition for the rule to be the best possible rule is that the compartment be completely autonomous. If the compartment is incompletely autonomous, a better rule can always be written, but at the expense of choosing as the automaton an enlarged system, including more compartments. If no such union of compartments is autonomous, a better rule can always be chosen, until finally the automaton is the complete community itself, with all its subcompartments. Thus, the above condition, to be called the predictivity condition, can be stated more generally: $A$ transition scheme incorporating variables associated only with a particular compartment (level) can only approach optimal predictivity to the extent that the compartment is autonomous. (A level of organization of a compartment is a compartment if it is the union of all the subcompartments at that level and in this case autonomy would be defined for the level and enlargement could correspond to addition of levels.)

Autonomy provides a necessary but not sufficient condition for a good rule because the initial state of the entire community is specified, not just of the compartment itself. If the rule is not good in this case, it could never be good if only the initial state of the compartment were specified. Alternatively, it must be good if the rule for the compartment is to be good. It would be possible to redefine transition schemes solely in terms of compartments (using only the initial states of the compartments). This would make it possible to formulate a sufficient condition, but would also generate many more conditional terms, representing the uncertainty in the behavior of the environment and the rest of the community given the behavior of the compartment.

\section{The Bath of Unrepresented Adaptabilities}

In and of itself the predictivity condition is basically trivial-all it says is that if other components give information about the compartment of interest, it is possible to construct a more predictive but less local transition scheme. However, in conjunction with the compensation equation (Equation 17) it is highly nontrivial and leads directly to the following key statement: $A s$ the predictive value of a transition scheme (transition function, rule, law) used to describe the behavior of any given compartment (level) of a global biological system depends less on the incorporation of variables associated with other compartments (levels) the adaptabilities of these other compartments (levels) becomes in general more important for main- 
taining this predictive value. This statement follows directly from the fact that predictivity means high independence, but high independence means that environmental disturbances affecting other compartments (levels) are absorbed and dissipated in those compartments or levels, with minimum ramification to the compartment (or level) of interest. There are four possibilities (assuming an uncertain environment):

(A) Independence high (i.e., comparable to modifiability)

(1) Low modifiability. In this case adaptabilities elsewhere in the system enable the compartment to support arbitrary but determinate and predictable dynamics despite environmental uncertainty, i.e., are unrepresented in these dynamics but are critical for supporting them.

(2) High modifiability. In this case the compartment of interest makes an efficient contribution to total adaptability, providing the anticipation term is relatively low (implying good anticipation). An optimal predictor can be constructed without reference to other compartments, but the best predictions are necessarily probablistic. If the transition scheme of the environment is added to the predictor it will be better, provided that the coordinated anticipation term is smaller than the modifiability, and can be deterministic to the extent that the anticipation term is small. If the adaptability of the compartment of interest is less than the total required adaptability (in general the case), adaptabilities unrepresented in its dynamics are critical for maintaining these dynamics.

(B) Independence low (smaller than the modifiability)

(3) Modifiability low. In this case adaptabilities elsewhere in the system absorb most of the environmental uncertainty, but nevertheless the modifications of these other compartments ramify to the compartment of interest, making its behavior less than optimally predictable without enlargement of the set of variables. Thus fewer unrepresented adaptabilities would play a role in supporting a predictable dynamics.

(4) Modifiability high. In this case the compartment of interest makes a less than maximally efficient contribution to total adaptability, providing the anticipation term is relatively low, but an optimal predictor cannot be constructed without enlargement of the set of variables, thereby implying that fewer unrepresented adaptabilities would play a role in supporting the (in general stochastic) dynamics.

As a somewhat contrived example, imagine a population undergoing phyletic evolution. In the case of low modifiability and high independence for the population level, all the uncertainties would be absorbed at the genetic and organismic levels. More realistically, these modifications would also appear in the population dynamics (the case of low modifiability and low independence). In the case of high modifiability and high independence, the lower level adaptabilities would not appear, but the population dynamics would itself contribute to the adaptability. A more realistic case is the one of high modifiability and low independence, in which case the genetic and organismic modifiabilities would manifest themselves 
at the population level, but the population dynamics would itself contribute to adaptability.

The conclusion is thus that any apparently autonomous compartment or level of biological organization is really highly controlled by a very much larger system of unviewed compartments or levels that are protecting it from environmental disturbance. It is important to recognize that this is not the same as saying that underlying the dynamics of, for example, a population, there are an enormous number of physiological and genetic processes, involving metabolism, repair, irritability, movement, and so forth. It is only in so far as these processes undergo either adaptive or maladaptive change that they can produce modifications in the dynamics of the population, and it is only in so far as any adaptive changes are unrealistically efficient that they can fail to produce modifications in these dynamics. In general the learning at one level can only rarely be completely hidden from the standpoint of the behavior of another.

The statements made in this section have been made for discrete time, finite state, probablistic dynamical systems. They are clearly also true for the deterministic case and could be generalized for continuous time and differentiable dynamical systems.

\section{Functional Significance of Stability Concepts}

\section{Role of the Represented Adaptabilities}

Now it is possible to address the question, what is the relation between the various forms of stability or instability exhibited by biological systems and their adaptability. According to the previous section, as the behavior of any particular level of biological organization appears more autonomous, the contribution of unrepresented adaptabilities at other levels becomes in general more critical for maintaining this behavior. The key to using hierarchical adaptability theory to study the functional significance of stability and bifurcation is to look at this statement from the other side, viz,, from the side of the represented adaptabilities. From this standpoint, as the behavior of any particular level of biological organization appears more autonomous, its potential contribution to maintaining the behavior patterns at other levels increases (though it can only make such a contribution if the modifiability term is large).

\section{Recovery of Stability Concepts}

Basic stability concepts (e.g., weak stability, asymptotic orbital stability, structural stability) are ordinarily used in biology within the context of differential models, whereas hierarchical adaptability theory has been formulated in a discrete time, finite state representation. Here the most convenient way to make crosscorrelation possible is to define analog concepts for the representation used here, basing these on the notion of a tolerance (originally discussed in the context of biological models by Zeeman ${ }^{9}$ and most prominantly developed for automaton 
systems by $\mathrm{M}$. Dal $\mathrm{Cin}^{10}$ ). This is a binary, symmetric, reflexive, but nontransitive relation on a set of states, to be denoted by " - " and which roughly corresponds to the idea of a (here arbitrarily established) allowable difference between states. Analog definitions are:

(A) Weak Stability. A trajectory of a deterministic transition scheme is weakly stable if and only if it is possible to define a nontrivial tolerance such that for all $n$ if $\alpha_{i j}{ }^{3}(t) \sim \alpha_{i j}{ }^{\prime}(t)$, then $\alpha_{i j}{ }^{\prime \prime}(t+n) \sim \alpha_{i j}{ }^{j}(t+n)$, where $\alpha_{i j}{ }^{\prime \prime}$ and $\alpha_{i j}{ }^{b}$ are the states at time $t+n$ and $\alpha_{i j}{ }^{\prime}$ and $\alpha_{i j}{ }^{3}$ are the states at time $t$. A scheme all of whose trajectories are weakly stable will be called weakly stable. Such schemes are analogous to differentiable dynamical systems with a constant of the motion (since a constant of the motion means that nearby states always remain nearby but never become identical).

(B) Strong Stability. A state $\alpha_{i j}{ }^{\prime}$ of a (probabilistic or deterministic) transition scheme is strictly stable if and only if it is possible to define a tolerance such that the system returns to $\alpha_{i j}{ }^{\prime}$ by time $t+n$ ( $n$ sufficiently large) if $\alpha_{i j}{ }^{\prime}(t)-\alpha_{i j}{ }^{\prime}(t)$, and furthermore if for all $k<n$ states at $t+n-k$ are either identical to or in tolerance with $\alpha_{i j}{ }^{\prime}$. Strong stability could also be defined for a trajectory if it is only required that by time $t+n$ the states are identical, but not necessarily identical to the initial state. For deterministic systems, strong stability is analogous to asymptotic orbital stability, where nearby states change to more and more nearby states (or where trajectories converge, as for stable limit cycles).

(C) Structural Stability. Consider the joint transition scheme $\omega_{p q} \omega_{r s}=$ $\left\{p\left(\alpha_{p q}{ }^{a}(t+1), \alpha_{r s}{ }^{b}(t+1) \mid \alpha(t), \beta(t)\right\}\right.$. The term $\alpha_{r s}{ }^{b}$ will be called a parameter (and $\omega_{r s}$ a parametric scheme) if it remains the same for all $t$, while $\alpha_{p q}{ }^{a}$ in general changes (i.e., is the variable). The joint scheme will be called structurally stable to a change from $\alpha_{r s}{ }^{a}$ to $\alpha_{r s}{ }^{c}$ if it is possible to define a tolerance on the states of compartment $(p, q)$ such that $\alpha_{p q}{ }^{\prime \prime}(t+n)-\alpha_{p q}{ }^{b}(t+n)$, where $n$ is arbitrary and the $\alpha_{p q}{ }^{\mu}$ are states of the compartment with parameter $\alpha_{p q}{ }^{a}$ and the $\alpha_{p q}{ }^{j}(t+n)$ are states of the compartment with parameter $\alpha_{p q}{ }^{c}$. If the scheme is not structurally stable to this particular change in parameter, the change will be called a bifurcation-producing change and the scheme will be said to bifurcate. The definition of structural stability is analogous to the dynamical notion of qualitative invariance to change in parameter and the notion of bifurcation to the notion of qualitative change in response to slight change in parameter. (If $\alpha_{p q}{ }^{\mu}(t+n)=\alpha_{p q}{ }^{g}(t+n)$, then rather than calling the joint scheme structurally stable it will be said that the two compartments do not interact.)

\section{Biology of Stability, Instability, and Bifurcation}

Stability and bifurcation play a fundamental role in relation to all the basic biological capabilities - metabolism, repair, growth, reproduction, irritability, and movement. Adaptability is also a fundamental biological capability, but in a higher order sense that it involves variation and modulation of these first order capabilities so as to absorb and dissipate disturbance. Here the concern is only with this connection, but in fact it is the decisive connection as regards the particular form stability and bifurcation take in relation to processes involving metabolism, 
repair, growth, and so forth. A major conclusion is that instability and bifurcation make a fundamental contribution to the stability of biological systems, or at least to the stability of the most essential processes in such systems.

To justify this statement, consider the fundamental biology of each of the basic notions of stability (from the standpoint of adaptability theory):

(A) Weak Stability. Weakly stable models are frequently criticized (as candidates for descriptions of biological systems) on the grounds that they are not structurally stable to a change in parameter that would represent the addition of even the smallest amount of dissipation. Nevertheless a compartment accurately describable by a weakly stable model would be capable of making a highly efficient contribution to adaptability and could under suitable conditions persist in a form so describable. The suitable condition is that the compartment be completely protected by a bath of adaptabilities provided by other compartments (possibly including its subcompartments or its supercompartments) from all disturbances that could introduce a source of dissipation into its dynamics. The efficiency of the contribution would result directly from this necessarily high autonomy together with the fact that slight perturbations (not introducing sources of dissipation) produce only slight modifications in the system and an enormous number of slight perturbations can therefore be absorbed without significant cost. The likelihood of the necessary conditions being met may not be compelling; nevertheless the above considerations should be taken into account if it appears that a system is accurately describable by a weakly stable model. (Examples of weakly stable models are the Lotka-Volterra equation, Kerner's" statistical mechanics of Lotka-Volterra systems, and Goodwin's ${ }^{12}$ statistical mechanics of gene-protein interactions in cells.)

(B) Strong Stability. The argument generally made in favor of strongly stable models is that they are necessarily dissipative (since they forget perturbation), and therefore not necessarily structurally instable to a perturbation that introduces more dissipation. The fundamental equations of physics are conservative, therefore weakly stable, so that ultimately the dissipation of disturbance is equivalent to the absorption of disturbance in a (weakly stable) heat bath. This heat bath is thus the unrepresented (physical) adaptability that supports the strongly stable dynamics of the compartment in question. However, the particular form of these dynamics is also maintained by other, biological adaptabilities, which are necessary to prevent different types of dissipative perturbation from mixing in, and which play an increasingly important role insofar as the degree of autonomy increases.

The above considerations suggest how strongly stable dynamics (with asymptotically stable states or limit cycle behavior) might be significant from the standpoint of a system performing particular biological functions, how the strong stability contributes to adaptability by representing the absorption of disturbances in a heat bath, and how the appearance of some degree of dynamical autonomy (or predictability in terms of a restricted set of variables) can more or less be supported by unrepresented adaptabilities. Taking the other point of view, however, instabilities of a strongly stable system (involving transitions among multiple steady states or multiple limit cycles) potentially provide adaptabilities that may be unrepresented in the dynamical descriptions of other compartments, but 
which support these dynamics. The costs of such adaptabilities depends on the biological structures and processes required, the modifications required to move from one stable state (organization) to another, degree of independence of such modifications from modifications of other compartments, and the compatibility of the different states with the efficiency of the compartment and other compartments (which might be expected to increase as the "nearness" of the states increases).

(C) Structural Stability. The argument for structurally stable models is the same as above, except that the dynamical structures can be so chosen that more or less wide classes of perturbation can be mixed in also so chosen that the bifurcations (catastrophes) occur in a structurally stable way. ${ }^{13,14}$ From the standpoint of adaptability there are three basic considerations, indicating both costs and advantages of structural stability and structural instability:

(1) Relation between independence and structurally stable behavior (stable or unstable). The states assumed by the varying compartment (i.e., the variable) are changed gradually as the states of parametric compartments (represented by the parameter) undergo changes. This means that modifications in different compartments are correlated, thereby apparently decreasing independence and increasing the cost of adaptability. Some caution is necessary, however, since the system is really more structurally stable if the "nearbyness" of the behavior of the variable compartment for different states of the parametric compartment increases, so that independence increases as structural stability increases provided the compartments interact. Indeed, another way of saying that the dynamics of a subcompartment of an interconnected system of compartments has high independence (or high degree of autonomy) is to say that these dynamics are structurally stable relative to changes in the other compartments (whose states may be lumped into parameters). To increase this independence (nearbyness of the behavior of a compartment despite possible change in the behavior of other compartments) the system must be built so that it is capable of supporting more modes of dissipation compatible with a given class of behaviors. This (along with structural constraint or internal decorrelation concomitant to weakened interaction) is the cost of independence. Furthermore, since independence has a basis in the construction of the system, it is itself an aspect of organization that must be protected from disturbance, implying that the whole hierarchy of adaptabilities contributes to the maintenance of the independence (i.e., structural stability or noninteraction) which increases their efficiency (self-consistency of the bootstrap principle).

(2) Contribution of structural stability of stable behavior. By structural stability of stable behavior is meant the structural stability away from the bifurcation points. Of two systems structurally stable to the same class of perturbations, the one which undergoes less quantitative change (therefore is more independent) is here called structurally stable. For the system less structurally stable (in this sense) adaptive or maladaptive changes in compartments (generally represented in terms of a single lumped parameter) causes any law written solely in terms of the variable of the compartment of original interest to be broken. However, the consequent quantitative changes in the compartment of interest potentially subserve the absorption of disturbance and therefore may also be adaptive, though in this case at a real increase in the cost of adaptability (because of the real decrease in 
independence). This cost can be reduced if the parametric compartment is organized in such a way that the modifications are really inexpensive. It can then be thought of as a sensor or control element and in fact low independence in this case increases adaptability by increasing anticipation (i.e., decreasing terms such as $H\left(\hat{\omega}_{p q} \mid \hat{\omega}^{\prime} \hat{\omega}_{r s}\right)$, where $\hat{\omega}_{r s}$ is the transition scheme of the parametric compartment). It might be noted that if the parametric compartment were excluded from consideration, the quantitative variation of behavior of the compartment of interest would appear as a form of unstable behavior.

(3) Contribution of structural stability of unstable behavior. By structural stability of unstable behavior is meant structural stability of the bifurcation process. Insofar as bifurcation allows for different modes of behavior (e.g., different modes of development), it provides a mechanism for absorbing disturbance, in just the same sense that weak stability or multiple steady states potentially absorb disturbance, except that the unstable behavior would appear quite incomprehensible if an attempt were made to describe it solely in terms of variables of the compartment of interest. As with the case for stable behavior, a decrease in independence would in general mean correlated modifications and therefore an increase in the cost of adaptability; but if the parametric compartment is specialized as an inexpensively modifiable sensor or controller, better anticipation is possible and it is more likely that a mode of behavior appropriate to the environment will be assumed.

In sum, the modifiability terms cross-correlate to instabilities, associated with jumps to alternate weakly or strongly stable states (absorption of disturbance) or to stable behavior involving the direct return to an initially strongly stable state subsequent to disturbance (dissipation of disturbance). The independence terms cross-correlate with either structural stability or weakening of interaction. The translations, however, are strictly applicable only to discrete time, finite state dynamical systems, although it is not unreasonable to expect essential carryover to more general situations.

\section{Conclusions}

Adaptability theory has a number of applications which have been described elsewhere, e.g., to homeostasis, ${ }^{3}$ patterns of adaptability in populations, ${ }^{\prime}$ routability of matter and energy flow in communities, ${ }^{15}$ and evolution of levels of organization. ${ }^{1}$ The discussion in the present paper, which deals with the crosscorrelation of the adaptability theory framework and dynamic biological models, also has evident implications for the construction and interpretation of dynamical models in biology.

A pertinent example is the long-standing issue as to the relation between complexity and stability of ecological systems. The elegant studies of May ${ }^{16}$ have clearly established that the probability of stability decreases as complexity-number and interdependence among species-increases. The basic reason is roughly that as the dimensionality of a space gets larger it becomes less likely to find multidimensional valleys and if such a valley is found it is more likely to be one which a small perturbation will cause the system to escape. Yet as May ${ }^{17}$ 
points out, complex communities in nature are not necessarily less stable than simple ones, and in fact climax communities are often quite complex. From the standpoint of adaptability theory the increase in complexity of the community level is quite compatible with the lowered stability, provided that adaptabilities unrepresented in the population dynamics are increased, either by increasing the modifability of subcompartments (increasing genetic and phenotypic plasticities) or by increasing the independence of the various forms of modifiability. In this way the community earns the advantages of complexity (e.g., specialization of labor) but without incurring the disadvantage of instability at the population level, essentially because the bath of unrepresented adaptabilities causes the environment it sees to appear quieter. However, it should also be remembered (from the standpoint of constructing models) that instability of the population dynamics may also make a contribution to adaptability, particularly if species can be stored (in spore or seed form) and if organisms are not too expensive to construct (e.g., microorganisms). Indeed adaptability theory suggests that as the environment is made harsher and more uncertain (thus increasing the requirements for adaptability and at the same time making it more difficult to pay for this adaptability) there will at some point be a switch between the two types of adaptability patterns. In either case to the issue of the relation between stability and complexity must be added the consideration of adaptability.

\section{REFERENCES}

1. ConRAD, M. 1972. Statistical and hierarchical aspects of biological organization. In Towards a Theoretical Biology. C. H. Waddington, Ed. Vol. 4: 189-221. Edinburgh University Press. Edinburgh.

2. Conrad, M. 1972. Can there be a theory of fitness? Intern. J. Neuroscience 3: 125-134.

3. Conrad, M. 1975. Analyzing ecosystem adaptability. Math. Biosci. 27:213-230.

4. Conrad, M. 1976. Biological adaptability: the statistical state model. Bioscience 27: 319-324.

5. Conrad, M. 1976. Patterns of biological control in ecosystems. In Systems Analysis and Simulation in Ecology. B. C. Patten, Ed. Vol. 4:431-456. Academic Press. New York.

6. ConRAD, M. 1977. Functional significance of biological variability. Bull. Math. Biol. 39: $139-156$.

7. Conrad, M. 1977. Biological adaptability and human ecology. In Proceedings of the First International Congress on Human Ecology. H. Knötig, Ed.: 467-473. Georgi Publishing Co. Switzerland.

8. Khinchin, A. I. 1957. Mathematical Foundations of Information Theory. Dover. New York.

9. Zeeman, E. C. \& O. P. Buneman. 1968. Tolerance spaces and the brain. In Towards a Theoretical Biology. C. H. Waddington, Ed. Vol. 1: 140-151. Edinburgh University Press. Edinburgh.

10. Dal CIN, M. 1974. Modifiable automata with tolerance: a model of learning. In Physics and Mathematics of the Nervous System. M. Conrad, W. Guttinger \& M. Dal Cin, Eds.: 442-458. Springer-Verlag. Heidelberg.

11. Kerner, E. 1957. A statistical mechanics of interacting biological species. Bull. Math. Biophys. 19:121-146.

12. Goodwin, B. C. 1963. Temporal Organization in Cells. Academic Press. New York, N.Y. 
13. Тном, R. 1970. Topological models in biology. In Towards a Theoretical Biology. C. H. Waddington, Ed. Vol. 1:95-120. Edinburgh University Press. Edinburgh.

14. Güttinger, W. 1974. Catastrophe geometry in physics and biology. In Physics and Mathematics of the Nervous System. M. Conrad, W. Guttinger \& M. Dal Cin, Eds.: 2-30. Springer-Verlag. Heidelberg.

15. Conrad, M. 1972. Stability of foodwebs and its relation to species diversity. J. Theoret. Biol. 34: 325-335.

16. MAY, R. M. 1975. Stability and Complexity in Model Ecosystems. 2nd edit. Princeton University Press. Princeton, N.J.

17. MaY, R. M. 1976. Patterns in multi-species communities. In Theoretical Ecology. R. M. May, Ed.: 136-152. Blackwell Scientific Publications. London. 